\title{
Aptamer-modified PLGA nanoparticle delivery of triplex forming oligonucleotide for targeted prostate cancer therapy
}

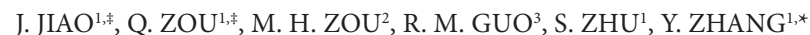

${ }^{1}$ Department of Nuclear Medicine, the Third Affiliated Hospital of Sun Yat-Sen University, 600 Tianhe Rd Guangzhou, China; ${ }^{2}$ Department of Ultrasonography, the Third Affiliated Hospital of Sun Yat-Sen University, 600 Tianhe Rd Guangzhou, China; Department of Radiology, the Third Affiliated Hospital of Sun Yat-Sen University, 600 Tianhe Rd Guangzhou, China

*Corrrespondence: zhangyong150825@163.com

${ }^{*}$ Contributed equally to this work.

Received November 24, 2015 / Accepted March 23, 2016

\begin{abstract}
Presented study aimed to prepare A10 aptamer-modified poly (D,L-lactic-co-glycolic acid) (PLGA) nanoparticles loaded with triplex forming oligonucleotides(TFO) for targeted prostate cancer therapy. We first synthesized a PLGA-PEG-Apt copolymer. The PLGA-PEG-Apt nanoparticles (NP-Apt) were loaded with TFO using double emulsion solvent evaporation method. Carboxy-fluorescein labeled TFO-NP-Apt, TFO-NP and TFO were prepared for cellular uptake experiments. Cell counting kit-8 (CCK-8) test was used to determine the ability of TFO-NP-Apt to inhibit LNCaP cell proliferation. RT-PCR and Western blot was conducted to analyze AR gene expressing. Then, a mouse model of prostate cancer was used to evaluate the anti-cancer effect of TFO-NP-Apt in vivo. We confirmed that the PLGA-PEG-Apt conjugation was successful. The TFO encapsulation efficiency and drug loading percentage were $46.1 \pm 3.6 \%$ and $40.8 \pm 5.3 \%$, respectively. TFO-NP-Apt showed a more efficient cellular uptake than TFO-NP or TFO in LNCaP cells. TFO-NP-Apt was significantly more cytotoxic than TFO-NP and TFO in the CCK-8 test $(p<0.001)$. TFO-NP-Apt silenced the AR gene better than unconjugated Apt, naked TFO, NP or saline. TFONP-Apt were more effective than TFO-NP, naked TFO, NP and saline at inhibiting prostate cancer growth in vivo $(p<0.05)$. Aptamer-modified TFO-loaded PLGA nanoparticles may prove useful in targeted therapy for advanced prostate cancer.
\end{abstract}

Key words: triplex forming oligonucleotide, aptamer, prostate-specific membrane antigen, prostate cancer

Prostate cancer is the second most common cancer and the sixth leading cause of cancer morbidity among males worldwide. [1] Current prostate cancer treatments are ineffective for advanced disease, which is treated by androgen ablation. Unfortunately, most patients who are treated with androgen ablation ultimately progress to lethal castration-resistant prostate cancer (CRPC). [2] Previous studies have found that androgen receptors (AR) are still expressed in CRPC and that $\mathrm{AR}$ signaling is involved in the progression of prostate cancer. The suppression of AR expression is thought to be a way to reduce tumor growth in AR-positive prostate cancers. [3]

We developed a triplex forming oligonucleotide (TFO) that was able to bind to AR gene in a sequence-specific manner. Our previous studies found that this TFO can suppress the expression of the AR gene and inhibit prostate cancer cell growth both in vitro and in vivo. [4] However, there are some challenges in delivering TFO to the targeted cells. It is necessary to find an optimal gene delivery system. Nanoparticles have been widely used for gene delivery due to their desired characteristics. However, the main obstacles to the use of such nanoparticles are enzymatic degeneration, a short half-life, and poor cellular uptake. [5] A nanoparticle with a special structure consisting of a gene, polymer matrix, biological stability group and targeting ligand, was found to be the ideal delivery system to overcome these obstacles. Poly (D,L-lactic-co-glycolic acid) (PLGA) controlled release systems are excellent choices for the clinic due to their safety. Poly (ethylene glycol) (PEG) prolongs the life of PLGA nanoparticles in the circulation, thereby enhancing permeability and retention (EPR) effects. However, PEG has been found to inhibit cellular uptake due to the shielding of surface charges. [6] The modification of this molecule with targeting ligands that specifically bind to the cell surface could potentially improve cellular uptake.

Aptamers (Apt) are short single-stranded oligonucleotides (DNA or RNA) that have specific 3D structure and are able to bind to target molecules with high affinity and specificity. 
[7] Because of their low molecular weight, lack of immunogenicity, and better availability, aptamers are widely used for targeted imaging and therapy. [8] In 2004, Farokhzad et al. were the first to conjugate aptamers to NP for the targeted delivery of drugs to cancer cells. [9] Since then, the feasibility of producing aptamer-modified NP has increased, with NPs becoming widely used for the delivery of targeted imaging and therapeutic agents. In this study, we aimed to develop TFO-encapsulated PLGA-PEG nanoparticles modified with the A10 aptamer that would target prostate specific membrane antigen (PSMA) before exploring the anti-cancer effect of the nanoparticles and the mechanism underlying this effect. With these goals in mind, we constructed a TFO delivery system using aptamer-modified PLGA-PEG nanoparticles.

\section{Materials and methods}

Poly (lactide-co-glycolide) (50/50) with terminal carboxylate groups (PLGA-COOH, inherent viscosity $0.20 \mathrm{dl} / \mathrm{g}$ in hexafluoroisopropanol, MW $20 \mathrm{kDa}$ ) was purchased from Chengdu Organic Chemicals Co., LTD., Chinese Academy of Science. NH2-PEG-COOH (MW 3400) was purchased from Yarebio (Shanghai, China). All of other the reagents were analytical grade and purchased from Sigma-Aldrich(St. Louis, MO, USA) unless otherwise noted. The RNA aptamer (sequence:5'-NH2-spacer-GGG/AGG/ACG/AUG/CGG/ AUC/AGC/CAU/GUU/UAC/GUC/ACU/CCU/UGU/CAA/ UCC/UCA/UCG/GCiT-30 with 2'-fluoro pyrimidines, a 5'amino group attached by a hexaethylene glycol spacer and a 3 '-inverted $\mathrm{T}$ cap) was synthesized by Integrated DNA technologies. The TFO- and FAM-labeled TFO (sequence: 5'-GGAGAGGAAGGAGGA-3') were synthesized by Sangon Biotech Co., LTD. (Shanghai, China). The LNCaP and PC3 prostate cancer cell lines were obtained from ATCC. The cell counting kit-8 (CCK-8) was obtained from Dojindo (Japan), while the RPMI 1640, OptiMEM, and fetal bovine serum (FBS) were obtained from Gibco BRL (Carlsbad, CA, USA). The AR antibody was obtained from Abcam.

Synthesis of PLGA-PEG-Apt. The PLGA-PEG-Apt was synthesized as previous described (Frank Gu, et al. 2008), In brieflyf, we synthesized PLGA-PEG-COOH. Then, we conjugated the RNA aptamer to the terminus of PLGA-PEG as follows. The PLGA-PEG-Apt was dissolved in $\mathrm{CDCl}_{3}$ and characterized by ${ }^{1} \mathrm{H}$ NMR at $400 \mathrm{~Hz}$ (AVANCE AV 400, Bruker, Switzerland) to determine its average molecular weight (Figure 1).

Preparation of TFO-loaded PLGA-PEG-Apt nanoparticles. TFO-loaded PLGA-PEG-Apt nanoparticles (TFO-Apt-NPs) were prepared using the water-in-oil-in-water $(\mathrm{w} 1 / \mathrm{o} / \mathrm{w} 2)$ double-emulsion solvent evaporation technique(Figure 2). Briefly, the TFO (8 OD) was mixed with spermidine at room temperature for $20 \mathrm{~min}$ on a rotary shaker to form a complex. One hundred microliters of TE buffer ( $\mathrm{pH}$ 8.0) containing 8 OD TFO were emulsified in 1\% (W/V) PLGA-PEG-Apt ethyl acetate (1 ml) using a homogenizer (Fluko, Shanghai, China) at $10000 \mathrm{rpm}$ for $1 \mathrm{~min}$. The primary emulsion was added dropwise into $2 \mathrm{ml}$ of $5 \%$ polyvinyl alcohol solution and homogenized for another $1 \mathrm{~min}$ to form the double emulsion. The double emulsion was added dropwise into $50 \mathrm{ml}$ of $1 \%$ PVA solution and agitated using a magnetic stirrer for $3 \mathrm{~h}$ at room temperature to remove the organic solvents. The NPs were purified by ultracentrifugation (Millipore, Billerica, USA) with a molecular mass cutoff of $10 \mathrm{kDa}$. The final NPs were suspended in nuclease-free water and stored at $4^{\circ} \mathrm{C}$.

Characterization of NPs. The characteristics of PLGAPEG-Apt were determined using nuclear magnetic resonance (NMR) spectroscopy. PLGA, PLGA-PEG, and PLGA-PEGApt were solubilized in $\mathrm{DCl}_{2}$ and analyzed in a $400 \mathrm{mHz}$ spectrometer (Varian, Palo Alto, CA).

Cellular uptake of FAM -labeled TFO-loaded nanoparticles in prostate cancer cells. To observe cellular uptake of TFO loaded nanopartilcles and the targeted ability of the nanoparticles, the LNCaP cells (PSMA+) and PC3 cells (PSMA-) were seeded on glass-bottom dishes $(15 \mathrm{~mm})$. A final concentration of $60 \mu \mathrm{mol} / \mathrm{L}$ FAM -TFO-loaded NP-Apt was added to the cells and incubated for different times, The LNCaP and PC3 cells were then washed with PBS three times, fixed with $4 \%$ paraformaldehyde, and stained with DAPI (KeyGEN BioTECH). Micrographs were then taken on a Confocal microscopy (ZEISS LSM 710). To quantitative analysis the uptake of nanoparticles, $10^{6} \mathrm{LNCaP}$ cells and PC3 cells were treated with $60 \mu \mathrm{mol} / \mathrm{L}$ FAM-labeled TFO-loaded NP-Apt for different times, respectively. The cells were washed 3 times with PBS, then measured with a confocal microscopy (ZEISS LSM 710).

Anti-cancer effect of TFO-NP-Apt in vitro. The anti-proliferative effect of TFO-NP-Apt on LNCaP cells was evaluated using a cell counting kit-8 (CCK-8, Dojindo, Japan). LNCaP cells were seeded in 96-microwell plates at a density of $1 \times 10^{4}$ cells per well and incubated at $37^{\circ} \mathrm{C}$ for 24 hours. The cells were then treated with up to $60 \mu \mathrm{mol} / \mathrm{L}$ TFO-NP-Apt for 72 hours. Ten microliters of CCK- 8 was subsequently added to each well, and the cells were incubated for another 2 hours. The anti-proliferative effects of the different drugs and different concentration were expressed as a curve.

Real-time RT-PCR analysis. Total RNA from cells was collected 72 hours after co-culture with TFO-NP-Apt, TFO$\mathrm{NP}, \mathrm{TFO}, \mathrm{NP}$ and saline and isolated using One-Step SYBR GreenER $^{\text {im }}$ Kit, (Thermo Fisher Scientific), and reverse transcribed according to manufacturer's instructions. Relative changes in gene expression were normalized to GAPDH RNA in the same cDNA sample.

Western blot analysis. To assess the expression of the AR gene in LNCaP cells, we transfected cells with TFO-NPApt, TFO-NP, TFO, NP and saline. The cells were harvested 72 hours post-transfection and lysed in cold RIPA buffer, and the lysate was incubated for 15 minutes at $4^{\circ} \mathrm{C}$ and centrifuged at $12,000 \mathrm{rpm}$ for 15 minutes. The protein concentration of the supernatant was determined using the Bradford assay (Bio-Rad, Hercules, CA). An equal amount (10 mg) of protein 
was subjected to electrophoresis on sodium dodecyl sulfatepolyacrylamide gels and transferred to polyvinylidene fluoride membranes (Millipore, Billerica, MA). The membranes were immunostained with antibodies specific to the AR protein (BioWorld, Visalia, CA). The blots were developed with enhanced chemiluminescence reagents (Amersham Pharmacia, Buckinghamshire, UK) and visualized with the Gene-Genius Imaging System (Syngene, Frederick, MD).

Anti-cancer effect of TFO-NP-Apt in vivo. We developed prostate cancer models through the s.c. injection of LNCaP cells into the flank of BALB/c nude mice. The anti-cancer effect of the different drugs was evaluated by the degree of tumor growth. After the tumors had developed to approximately $250 \mathrm{~mm}^{3}$, we divided the mice into five groups $(n=7)$ as follows: TFO-NP-Apt, TFO-NP, TFO, NP, and saline. The groups were designed to minimize body weight and tumor size differences among the subjects. Every mouse was administered $5 \mathrm{mg} / \mathrm{kg}$ of the designated drug every two days. Tumor size and body weight were then monitored for three months.

Statistical analysis. The data are presented as means \pm standard deviation. Statistically significant differences were determined using two-sample $t$-tests and analysis of variance, with $P \leq 0.05$ as the significance level.

\section{Results}

Characterization of the PLGA-PEG-Apt copolymer. To increase hydrophilicity and reduce nonspecific interactions
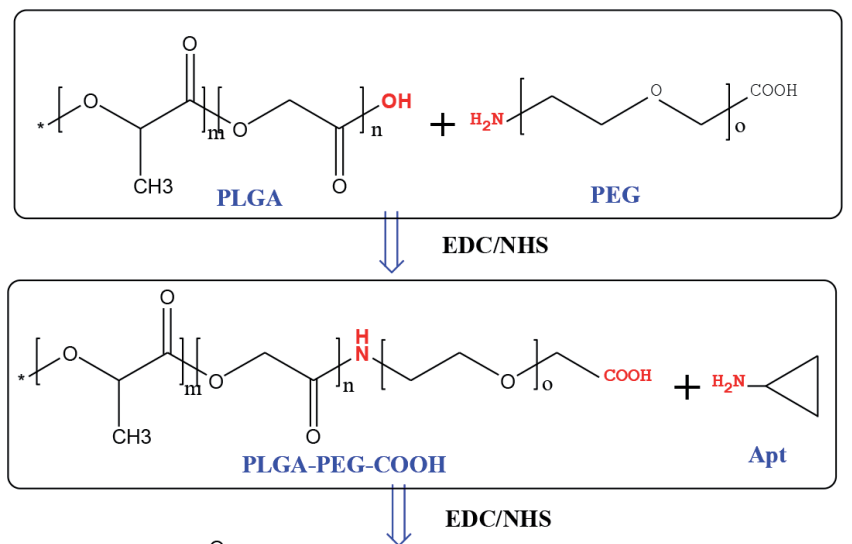<smiles>CCCCCCCCCC(C)NC(C)(C)C(=O)COC(C)C(=O)OC(C)(C)C</smiles>

Figure 1. The PLGA-PEG-Apt was synthesized in two steps: first, we synthesized PLGA-PEG-COOH. Then, we conjugated the RNA aptamer to the terminus of PLGA-PEG.

with the cellular membrane, we used PEG as a spacer between PLGA and the aptamer. The $\mathrm{DCl}_{2}$ solvent peak was found at 4.65 ppm (Figure 3). The PLGA-PEG NMR spectrum had multiple peaks corresponding to the repeat units in PEG at $3.5 \mathrm{ppm}$, confirming the conjugation of PEG to PLGA. The PLGA-PEG-Apt NMR spectrum exhibited multiple peaks

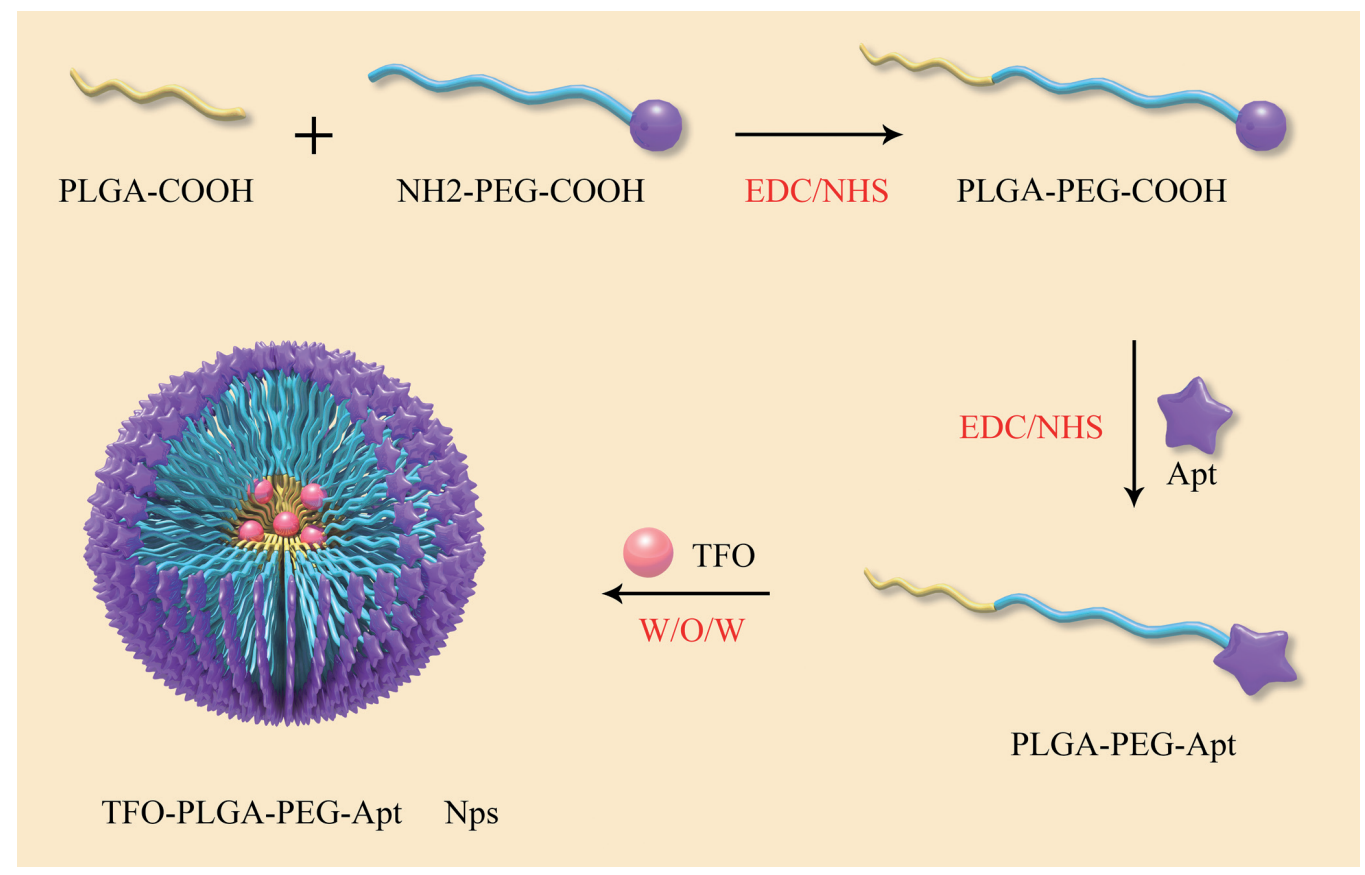

Figure 2. Preparation of aptamer-functionalized TFO-loaded nanoparticles (TFO-NP-Apt). TFO was encapsulated in the PLGA-PEG-Apt nanoparticles using an emulsion/solvent evaporation method. 


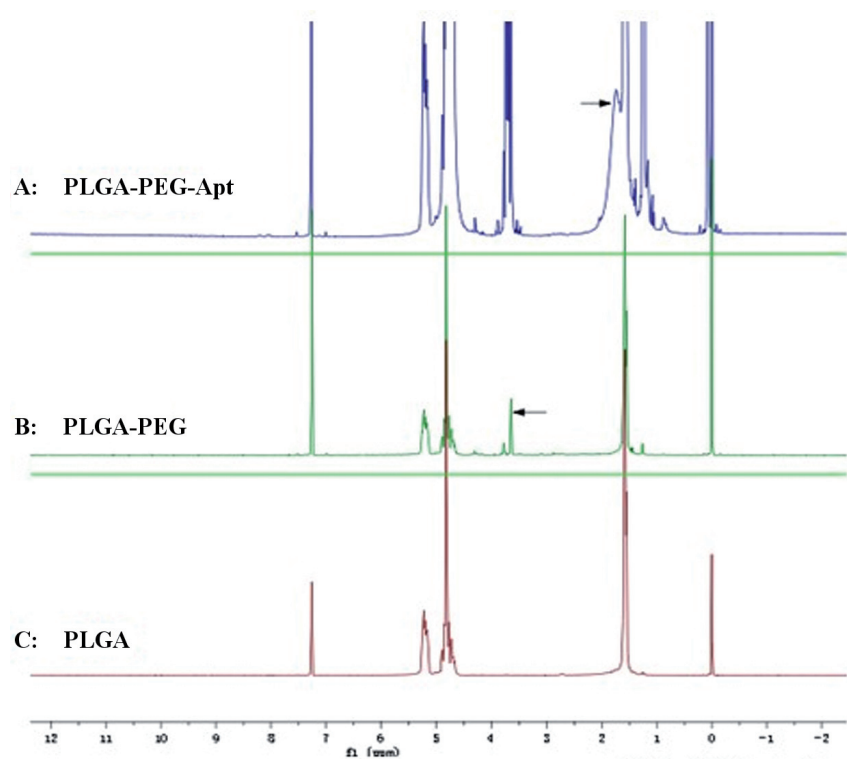

Figure 3. ${ }^{1} \mathrm{H}$ NMR characterization of PLGA, PLGA-PEG and PLGAPEG-Apt.

corresponding to the repeat units in Apt between 1.8-2.1 ppm, confirming the conjugation of Apt to PLGA-PEG.

Characterization of TFO-PLGA-PEG-Apt nanoparticles. The sizes and zeta potentials of the TFO-NP-Apt nanoparticles were analyzed using the Zetasizer Nano-ZS90. As shown in
Figure 4, the average size of the TFO-NP-Apt nanoparticles was $227.1 \pm 7.8 \mathrm{~nm}$ and the zeta potential was $-35.5 \pm-3.3 \mathrm{mv}$ (mean $\pm \mathrm{SD}, \mathrm{n}=3$ ). The SEM graphs indicate that the nanoparticles appear to be round, with smooth surfaces and a uniform size distribution.

Drug encapsulation efficiency and loading capacity. The encapsulation of TFO in TFO-NP and TFO-NP -Apt was $46.1 \pm 3.6 \%$ and $40.8 \pm 5.3 \%(n=3)$, respectively. The loading percentage of TFO-NP and TFO-NP -Apt was $4.23 \pm 0.21 \mu \mathrm{g} /$ $\mathrm{mg}$ and $3.35 \pm 0.16 \mu \mathrm{g} / \mathrm{mg}$, respectively $(n=3)$.

Uptake of FAM-labeled TFO nanoparticles by prostate cancer cells. The uptake of FAM-labeled TFO by LNCaP cells and PC3 cells was determined using confocal microscopy and quantified by determining the percentage of FAM positive cells. After the exposure of LNCaP cells and PC3 cells to FAM-labeled TFO-loaded NP-Apt $(60 \mu \mathrm{mol} / \mathrm{L})$ for different times $(30 \mathrm{~min}$, $120 \mathrm{~min}$ ), the LNCaP cells (PSMA+) group exhibited significantly higher uptake efficiency than the PC3 cells (PSMA-) groups at both time points (30min, $120 \mathrm{~min}$ ) (Figure 5).

Anti-proliferation assay. To evaluate the anticancer potential of TFO-NP-Apt, TFO-NP, and TFO, we performed a series of in vitro cytotoxicity assays using the CCK8 assay. LNCaP cells were treated with different concentrations of TFO-NP-Apt, TFO-NP, TFO, and saline. The results show that cell growth was inhibited in a dose-dependent manner (Figure 6), with the anti-proliferative abilities ordered as follows: TFO-NP-Apt $>$ TFO-NP>TFO.

AR mRNA and protein expression. First, we used RT-PCR to detect the mRNA expression of AR, and found that TFO-

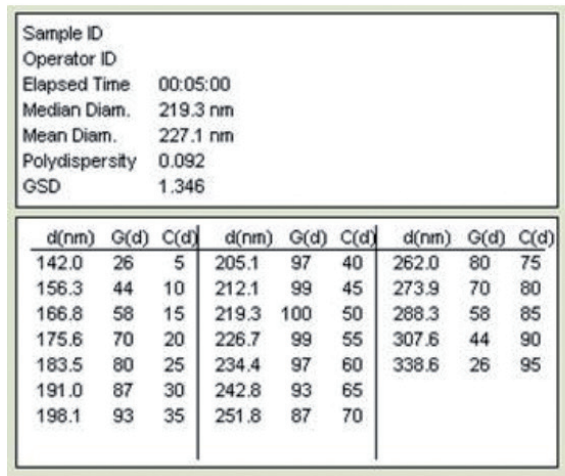
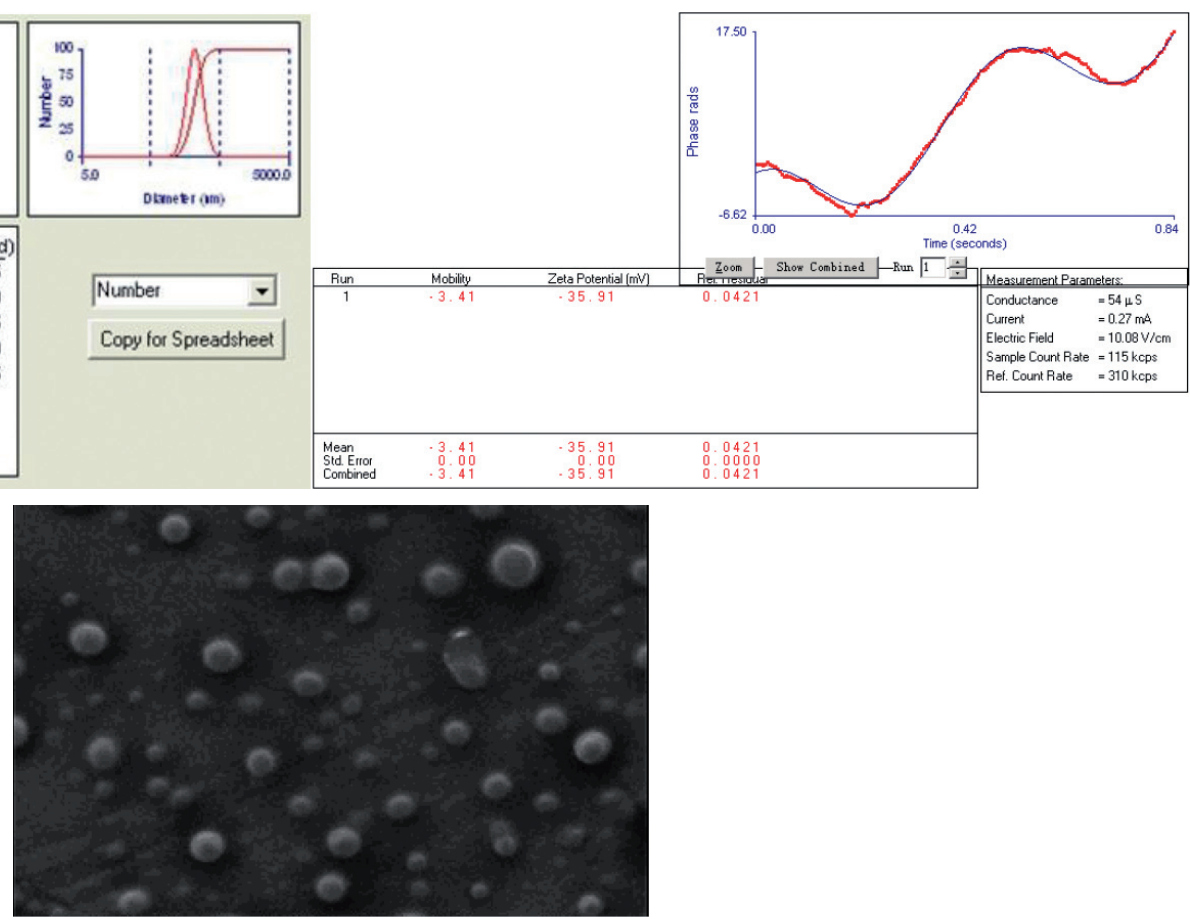

Figure 4. Size distribution, morphology and zeta potential of nanoparticles 
$\mathbf{A}$

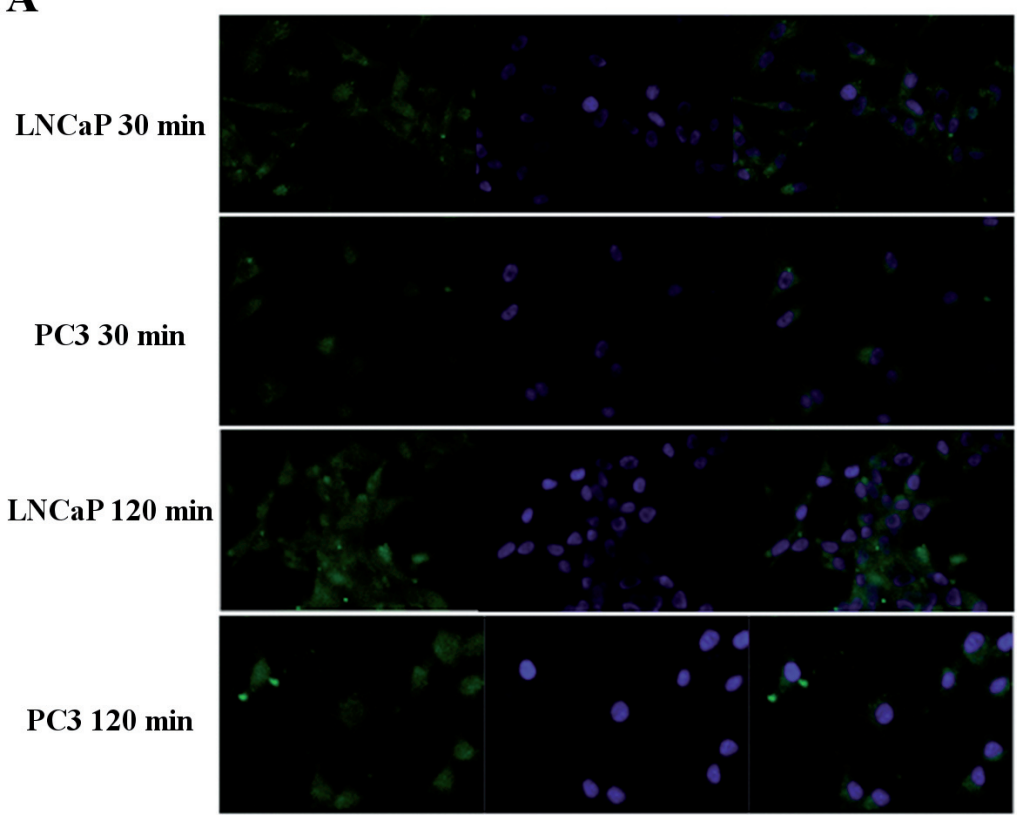

B

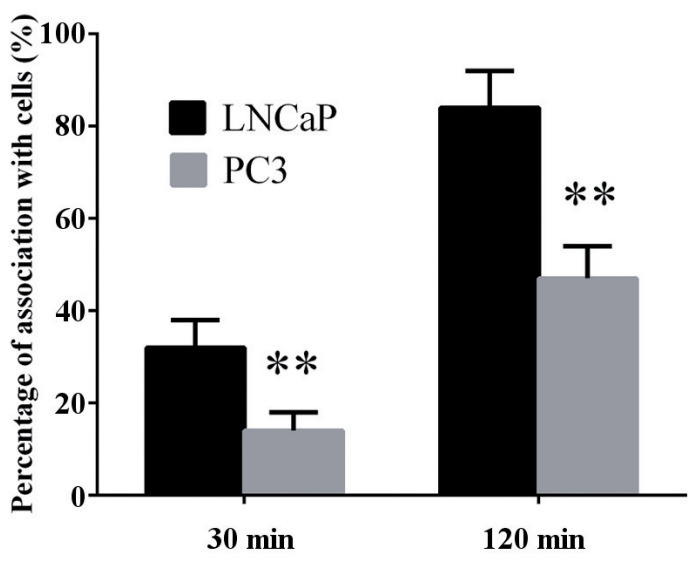

Figure 5. Confocal microscopy images (400X) and fluorescent-activated cell sorting analysis of LNCaP cells (PSMA+) and PC3 cells (PSMA-) after a $30 \mathrm{~min}$ and $120 \mathrm{~min}$ incubation with FAM-labeled TFO PLGA-PEG-Apt. A (fluorescence) and B (quantitative expression). The results are shown as a function of concentration.

NP-Apt, TFO-NP, and TFO all had the ability to decrease the expression of the AR mRNA, with the effects being ordered as follows: TFO-NP-Apt $>$ TFO-NP $>$ TFO $>\mathrm{NP}>$ saline. Further, we also examined the AR protein expression, and results showed that the protein level was also downregulated, which was consistent with the mRNA analysis. We also found that the TFO-mediated downregulation of the AR gene decreases cell viability. This finding indicates that the TFO-NP-Apt can effectively carry TFO into cells to have an inhibitory effect on the proliferation of prostate cancer cells (Figure 7).

Anti-cancer effect of TFO-NP-Apt in vivo. The anticancer effect of TFO-NP-Apt in vivo was determined using $\mathrm{BALB} / \mathrm{c}$ nude mice bearing a $\mathrm{LNCaP}$ cell xenograft. The mice were intravenously administered TFO-NP-Apt, TFO-NP, TFO, NP, or saline every two days $(5 \mathrm{mg} / \mathrm{kg}$ ). The tumor size and body weight of the mice were measured for three months. Our results showed that the intravenous administration of TFO-NP-Apt was significantly more efficacious than TFO-NP, TFO, NP, and saline at inhibiting tumor growth. The results demonstrated that A10 aptamer-modified PLGA nanoparticles ensured more TFO uptake by PSMA positive prostate cancer cells. The PLGA-PEG and saline groups exhibited no tumor regression (Figure 8).

\section{Discussion}

In this study, we targeted successfully prostate cancer cells with A10 aptamer-modified PLGA nanoparticles. The nanoparticles we prepared can effectively deliver TFO to prostate cancer cells, which leads to cell viability decrease and may be useful in the treatment of advanced prostate cancer. The PLGA nanoparticles we prepared effectively delivered water soluble TFO to prostate cancer cells overexpressing PSMA, resulting in tumoricidal effects as verified by cell viability assays. Our results suggest that aptamer-conjugated PLGA nanoparticles represent a novel therapy for advanced prostate cancers.

PLGA has become widely used for drug delivery because of its biodegradable and biocompatible nature. [10] Our previous work suggested that TFO could inhibit prostate cancer cell growth and that the loading of PLGA nanoparticles with

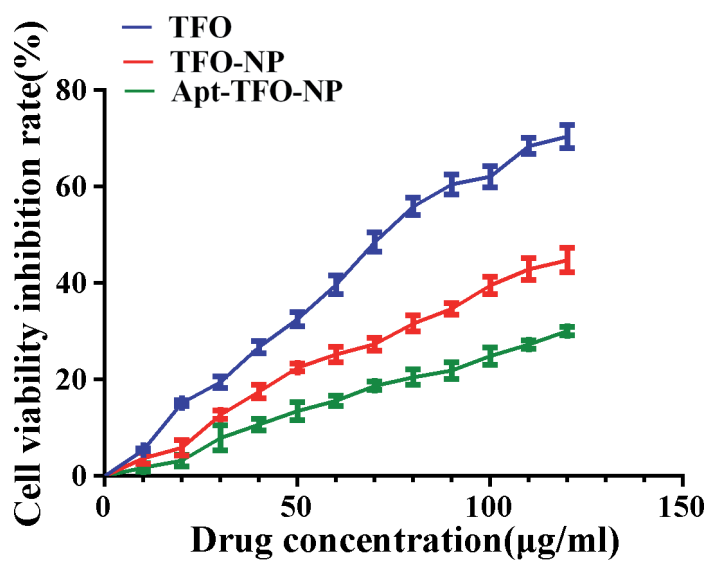

Figure 6. Cell viability of LNCaP cells treated with TFO-NP-Apt, TFO-NP, and naked TFO. 
A

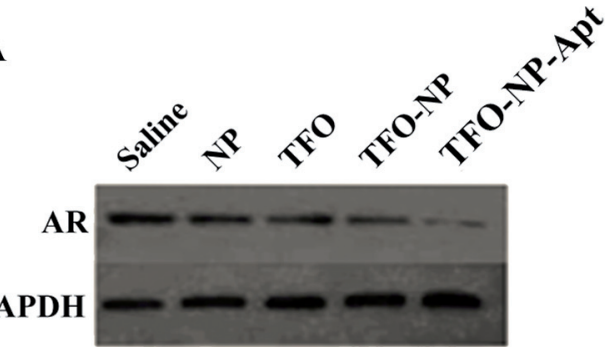

B

C
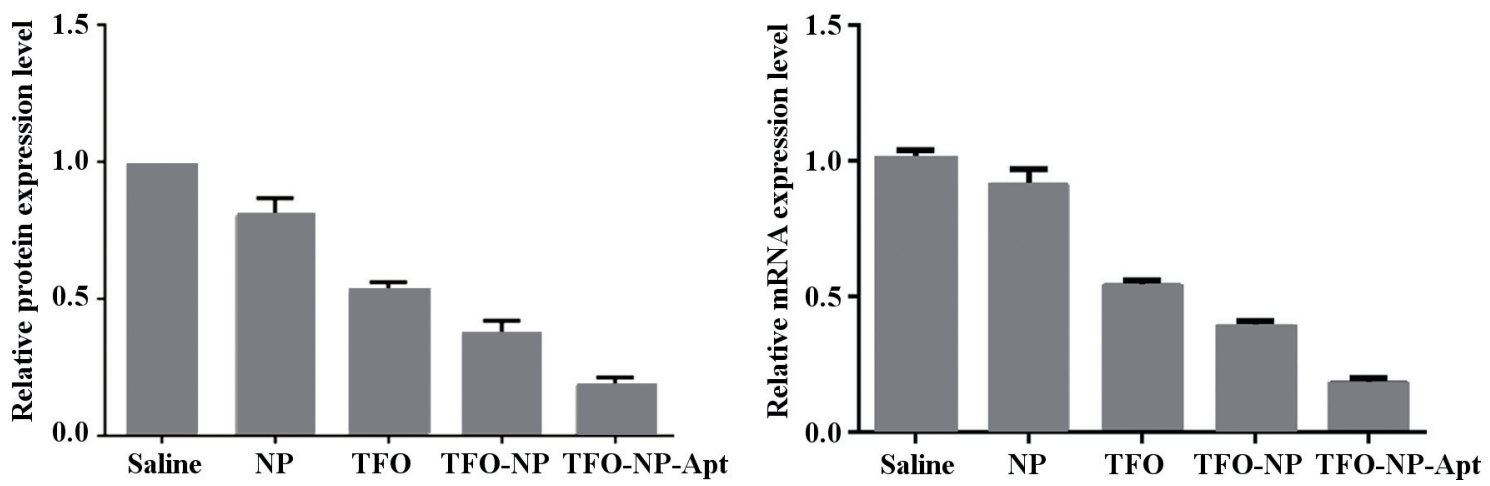

Figure 7. Western blot $(A, B)$ and Real-time RT-PCR (C) analysis of LNCaP cells after the administration of different drugs.

TFO could increase these effects. In addition, the surface of PLGA nanoparticles can be modified with targeting moieties such as antibodies, proteins, and small molecules. [11] The present study found that the A10 aptamer-modified PLGA nanoparticles target to prostate cancer cells and are more effective at silencing the expression of the AR gene and decreasing cell viability than other treatments. We also found that the particle size, zeta potential and loading efficiency of TFO were not obviously changed, which is of great value for future clinical use. Aptamers are widely used for targeted imaging and therapy. $[12,13]$ The A10 aptamer was the first aptamer to be conjugated with nanoparticles for the targeting of cancer, and has been widely used for targeted chemotherapy and imaging. In this study, we conjugated the A10 aptamer to the PLGAPEG copolymer and then to formulated nanoparticles loaded with water soluble TFO. This method improved the targeting and uptake of TFO.

AR silencing has been proven as an effective therapy for advanced prostate cancer. $[14,15]$ siRNA and antisense mRNA are routinely used for AR silencing. TFO binds to the
A

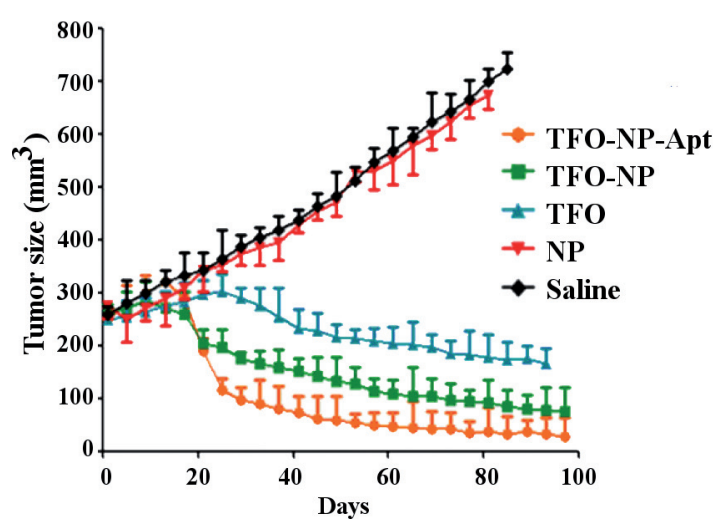

B

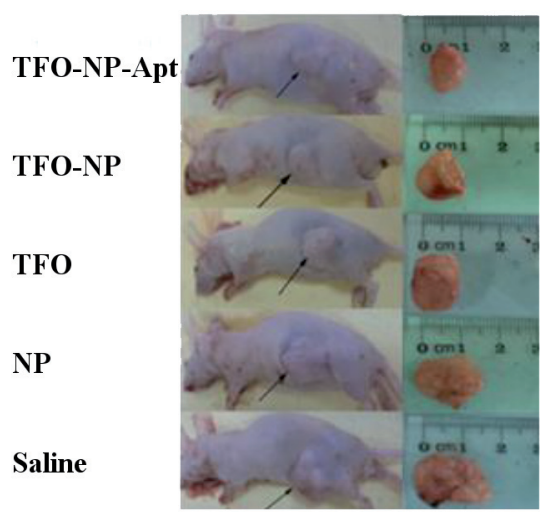

Figure 8. Intravenous injection of TFO-NP-Apt in vivo inhibited tumor growth. (A)Tumor growth curves for BALB/c nude mice bearing LNCaP cell xenografts after the intravenous administration of TFO-NP-Apt, TFO-NP, TFO, NP, and saline. (B) Actual sizes of representative tumors. 
AR gene in a sequence-specific manner, which prevents AR gene expression at the translation stage. Our previous study proved the anti-prostate cancer effects of TFO. In this study, we improved the TFO loading methods and targeted prostate cancer cells using aptamer-modified nanoparticles to enhance the uptake of TFO.

In conclusion, we effectively prepared TFO-loaded PLGA nanoparticles for targeted prostate cancer therapy. Our results demonstrate that the loading of PLGA nanoparticles with TFO can effectively silence AR gene expression and inhibit prostate cancer cell growth. The A10 aptamer enhanced the targeting ability of PLGA nanoparticles, which could potentially enhance the therapeutic efficacy and decrease the toxic side effects of current treatments.

Acknowledgments: We acknowledge the financial support from the National Natural Science Foundation of China (Project No. 30970857) and the key projects of Nature science foundation of Guangdong province (Project No.8251008901000009)

\section{References}

[1] SIEGEL R. andNAISHADHAM D., JEMAL A. Cancer statistics, 2013. CA Cancer J Clin. 2013; 1: 11-30. http://dx.doi. org/10.3322/caac. 21166

[2] SCHER H. I., Sawyers C. L. Biology of progressive, castrationresistant prostate cancer: directed therapies targeting the androgen-receptor signaling axis. J Clin Oncol. 2005; 32: 8253-8261. http://dx.doi.org/10.1200/JCO.2005.03.4777

[3] YANG J., XIE S. X., HUANG Y., LING M., LIU J., et al. Prostate-targeted biodegradable nanoparticles loaded with androgen receptor silencing constructs eradicate xenograft tumors in mice. Nanomedicine (Lond). 2012; 9: 1297-1309. http://dx.doi.org/10.2217/nnm.12.14

[4] ZHANG Y., MA Y., LU H. P., GAO J. H., LIANG C. S., et al. Inhibition of human prostate cancer xenograft growth by 125I labeled triple-helix forming oligonucleotide directed against androgen receptor. Chin Med J (Engl). 2008; 22: 2284-2289.

[5] ZHANG L., FENG Q., WANG J., SUN J., SHI X., et al. Microfluidic synthesis of rigid nanovesicles for hydrophilic reagents delivery. Angew Chem Int Ed Engl. 2015; 13: 3952-3956. http://dx.doi.org/10.1002/anie.201500096

[6] BAYSAL I., YABANOGLU-CIFTCI S., TUNC-SARISOZEN Y., ULUBAYRAM K., UCAR G. Interaction of selegilineloaded PLGA-b-PEG nanoparticles with beta-amyloid fibrils.
J Neural Transm. 2013; 6: 903-910. http://dx.doi.org/10.1007/ s00702-013-0992-2

[7] GAO L., LI Q., LI R., YAN L., ZHOU Y., et al. Highly sensitive detection for proteins using graphene oxide-aptamer based sensors. Nanoscale. 2015; 25: 10903-10907. http://dx.doi. org/10.1039/C5NR01187F

[8] CHEN W., ZENG W., SUN J., YANG M., LI L., et al. Construction of an Aptamer-SiRNA Chimera-Modified Tissue-Engineered Blood Vessel for Cell-Type-Specific Capture and Delivery. ACS Nano. 2015; 6: 6069-6076. http://dx.doi. org/10.1021/acsnano.5b01203

[9] FAROKHZAD O. C., JON S., KHADEMHOSSEINI A., TRAN T. N., LAVAN D. A., et al. Nanoparticle-aptamer bioconjugates: a new approach for targeting prostate cancer cells. Cancer Res. 2004; 21: 7668-7672. http://dx.doi.org/10.1158/0008-5472. CAN-04-2550

[10] MALATHI S., Nandhakumar P., Pandiyan V., Webster T. J., Balasubramanian S. Novel PLGA-based nanoparticles for the oral delivery of insulin. Int J Nanomedicine. 2015; 2207-2218.

[11] LI L., XIANG D., SHIGDAR S., YANG W., LI Q., et al. Epithelial cell adhesion molecule aptamer functionalized PLGA-lecithin-curcumin-PEG nanoparticles for targeted drug delivery to human colorectal adenocarcinoma cells. Int J Nanomedicine. 2014; 1083-1096.

[12] DASSIE J. P., LIU X. Y., THOMAS G. S., WHITAKER R. M., THIEL K. W., et al. Systemic administration of optimized aptamer-siRNA chimeras promotes regression of PSMAexpressing tumors. Nat Biotechnol. 2009; 9: 839-849. http:// dx.doi.org/10.1038/nbt.1560

[13] NI X., ZHANG Y., RIBAS J., CHOWDHURY W. H., CASTANARES M., et al. Prostate-targeted radiosensitization via aptamer-shRNA chimeras in human tumor xenografts. J Clin Invest. 2011; 6: 2383-2390. http://dx.doi.org/10.1172/ JCI45109

[14] AZUMA K., NAKASHIRO K., SASAKI T., GODA H., ONODERA J., et al. Anti-tumor effect of small interfering RNA targeting the androgen receptor in human androgenindependent prostate cancer cells. Biochem Biophys Res Commun. 2010; 1: 1075-1079. http://dx.doi.org/10.1016/j. bbrc.2009.12.024

[15] SUN A., TANG J., TERRANOVA P. F., ZHANG X., THRASHER J. B., et al. Adeno-associated virus-delivered short hairpin-structured RNA for androgen receptor gene silencing induces tumor eradication of prostate cancer xenografts in nude mice: a preclinical study. Int J Cancer. 2010; 3: 764-774. http://dx.doi.org/10.1002/ijc.24778 